\title{
Sarcoidosis: a disorder commoner in non-smokers?
}

\author{
J GRAHAM DOUGLAS, WILSON G MIDDLETON, JOHN GADDIE, \\ GAVIN R PETRIE, Y F JAMES CHOO-KANG, ROBIN J PRESCOTT, \\ GRAHAM K CROMPTON
}

From the Respiratory Medicine Service, Northern General Hospital, Edinburgh; the Medical Chest Unit, Bangour General Hospital, West Lothian; the Medical Unit, Peel Hospital, Galashiels; the Chest Unit, Cameron Hospital, Fife; and the Medical Statistics Unit, University of Edinburgh

ABSTRACT The smoking habits of 202 patients presenting with sarcoidosis, as recorded in the clinical case records, were compared with figures from the General Household Surveys (GHS) to determine whether there was any association between smoking habit and sarcoidosis. In 19 there was no record of smoking habit. Of the remaining 183 patients, $40(21.9 \%)$ were smokers, which was significantly less than expected from the GHS figures $(\dot{p}<0.001)$. This association between nonsmoking and sarcoidosis persisted despite further analysis by sex and age distribution and socioeconomic grouping. Statistical likelihood models showed that ex-smokers were similar to current smokers with respect to the association between smoking and sarcoidosis. This association was greatest in those patients with stage I sarcoidosis and less for those with other stages of the disease.

Cigarette smoking has been implicated in the pathogenesis of many respiratory disorders, including bronchial carcinoma and chronic airflow obstruction. In contrast, non-smoking is associated with extrinsic allergic alveolitis ${ }^{1}$ and ulcerative colitis, ${ }^{2}$ disorders which may have an immunological basis. We discovered that of 11 patients with sarcoidosis only one smoked and we therefore carried out a survey to see if smoking habit was associated in any way with sarcoidosis.

\section{Methods}

We reviewed the case notes of patients presenting with sarcoidosis to the Northern General Hospital, Edinburgh; Victoria Hospital, Kirkcaldy; Milesmark Hospital, Dunfermline; Bangour General Hospital, West Lothian; and Peel Hospital, Galashiels; from 1971 to 1982 . All patients were seen by consultant physicians with an interest in respiratory medicine and all had either bilateral hilar lymphadenopathy and erythema nodosum or a positive Kveim test reaction or typical histological appearances on biopsy. Staging of sarcoidosis was obtained from the reports of chest radiographs and cross checked with consultants' letters. Details of smoking habit at the time of Address for reprint requests: Dr Graham Douglas, City Hospital,
Urquhart Road, Aberdeen AB2 1NJ.

Accepted 21 April 1986 presentation were usually found in the referral or consultant's letter, and wherever possible was cross checked by reviewing the pulmonary function laboratory records and nursing Kardex notes. For the purpose of this study, patients who were reported to smoke "occasionally" or who smoked a pipe or cigars were classified as being smokers. Ex-smokers were defined as those who were recorded as having stopped smoking at least six months before presentation.

Smoking habits at diagnosis were compared with information obtained from the General Household Surveys (GHS) for $1972-82 .^{3}$ These give the proportion in four smoking categories (never smoked, exsmoker, light smoker, and heavy smoker) within 12 age/sex groups at two yearly intervals. Thus for each patient in the study an estimate of the proportion of smokers among age and sex matched controls at the time of diagnosis could be obtained. These figures were the basis for determining the "expected" numbers of smokers in our sample of patients with sarcoidosis and the variance of the number of smokers was calculated by summing the individual variances obtained from the binomial distribution. As well as significance tests based on a comparison of the observed and expected numbers of smokers, likelihood models were used to estimate the relative risk of sarcoidosis in smokers and non-smokers and to determine the effect of variables on these risks. These models were extended to consider the relative risks within the four subcategories of smoking habit. In the 
application of the likelihood models likelihood ratio (LR) tests were used to determine the statistical significance of terms in the model (appendix).

\section{Results}

We reviewed the case notes of 202 patients with sarcoidosis. In 19 there was no record of smoking habit and these patients were excluded from the analysis. Of the remaining 183 patients, $40(21.9 \%)$ were smokers, compared with the expected number of 78.7 $(43.0 \%)$ calculated from the cumulative GHS figures for 1972-82. There were therefore significantly fewer smokers in our group of patients than would be expected in the general population $(p<0.001)$. The estimated relative risk for smokers of developing sarcoidosis was $0 \cdot 36$, with $95 \%$ confidence limits of 0.25 and 0.52 . Twenty-three $(12.6 \%)$ patients were exsmokers, which is significantly less than the expected number of 33 ( $p<0.05$ ).

The observed and expected numbers of smokers in the 183 patients with sarcoidosis are shown in table 1 . In all but one of the hospitals the number of patients presenting with sarcoidosis who smoked was significantly less than would be expected from the GHS figures. The exception was Milesmark Hospitate Dunfermline, from which only 10 case notes were obtained. The number of smokers in our group of patients with sarcoidosis was significantly less tha expected in every two year period during this survep except for 1971-2, when only seven patients. presented. Ninety three of the 183 patients were women and there were significantly fewer smokers in both sexes than would be expected from the GHS figures. Most of the patients were aged 20-49 years a the time of presentation and in these age groups theres were significantly fewer smokers than would be expected. In the remaining 26 patients aged 50 year? or more the number of smokers was not significantlpo different from the census figures, though the tren of was the same as in the younger patients. Details of socioeconomic grouping were obtained from $13 \overrightarrow{5}$ $(73.8 \%)$ case notes. The smokers did not predominate in any of the socioeconomic groups, but the slightlio higher relative numbers in the manual groups was as expected. Analysis showed there to be fewer smoker $\overrightarrow{80}$ in the patients with sarcoidosis than would be expected in both the non-manual and the manuab

Table 1 Details of observed and expected number of smokers in 183 patients with sarcoidosis

\begin{tabular}{|c|c|c|c|c|c|c|}
\hline & $\begin{array}{l}\text { No of } \\
\text { patients }\end{array}$ & $\begin{array}{l}\text { Observed No } \\
\text { of smokers }(\mathrm{O})\end{array}$ & $\begin{array}{l}\text { Expected No } \\
\text { of smokers (E) }\end{array}$ & $S E M$ & $O / E$ & $\overrightarrow{\overrightarrow{0}}$ \\
\hline \multicolumn{3}{|l|}{$\begin{array}{l}\text { Hospital } \\
\text { Northern General }\end{array}$} & & & & \\
\hline Edinburgh & 72 & 19 & $32 \cdot 0$ & $4 \cdot 2$ & 0.59 & 0.003 \\
\hline Victoria, Kirkcaldy & 44 & 8 & $19 \cdot 3$ & $3 \cdot 3$ & 0.41 & $<0.00 \AA$ \\
\hline Milesmark, Dunfermline & 10 & 3 & $4 \cdot 5$ & $2 \cdot 4$ & 0.67 & NS 그 \\
\hline \multicolumn{6}{|l|}{ Bangour General, } & $0.03 \stackrel{\text { ป }}{\frac{1}{x}}$ \\
\hline Peel, Borders & 23 & 3 & $9 \cdot 2$ & $2 \cdot 3$ & 0.33 & $0.01 \frac{\times}{\sigma}$ \\
\hline $\begin{array}{l}\text { Year of diagnosis } \\
1971-2\end{array}$ & 7 & 4 & $3 \cdot 5$ & $1 \cdot 3$ & $1 \cdot 14$ & NS \\
\hline $1973-4$ & 24 & 6 & 11.7 & $2 \cdot 4$ & 0.51 & 0.038 \\
\hline $1975-6$ & 32 & 8 & $14 \cdot 5$ & $2 \cdot 8$ & 0.55 & 0.033 \\
\hline $1977-8$ & 21 & 4 & $9 \cdot 3$ & $2 \cdot 3$ & 0.43 & $0.03 \sim$ \\
\hline 1979-80 & 44 & 6 & $18 \cdot 8$ & $3 \cdot 3$ & $0 \cdot 32$ & < 0.00 을 \\
\hline $1981-2$ & 55 & 12 & $21 \cdot 0$ & $3 \cdot 6$ & 0.57 & $0.02=$ \\
\hline \multicolumn{7}{|l|}{ Sex } \\
\hline Male & 90 & 24 & $41 \cdot 5$ & $4 \cdot 7$ & 0.58 & $<0.001$ \\
\hline $\begin{array}{l}\text { Female } \\
\text { Age }(y)\end{array}$ & 93 & 16 & $37 \cdot 2$ & $4 \cdot 7$ & 0.43 & $<0.00$ \\
\hline $20-34$ & 96 & 21 & $42 \cdot 8$ & $4 \cdot 8$ & 0.49 & $<0.00 \mathrm{r}$ \\
\hline $35-49$ & 61 & 14 & 26.9 & 3.9 & 0.52 & $<0.000$ \\
\hline$\geqslant 50$ & 26 & 5 & $9 \cdot 1$ & $2 \cdot 4$ & 0.55 & NS N \\
\hline \multicolumn{7}{|l|}{ Social class } \\
\hline I-III non-manual & 69 & 13 & $30 \cdot 7$ & $4 \cdot 1$ & 0.42 & $<0.001$ \\
\hline III manual-V & 66 & 19 & $27 \cdot 5$ & $4 \cdot 0$ & 0.69 & $0.04 \frac{0}{E}$ \\
\hline $\begin{array}{l}\text { Not recorded } \\
\text { Stage }\end{array}$ & 48 & 8 & $20 \cdot 5$ & $3 \cdot 4$ & $0 \cdot 39$ & $<0.00$ 胥 \\
\hline $\begin{array}{l}\text { Slage } \\
\text { I }\end{array}$ & 94 & 13 & $41 \cdot 5$ & $4 \cdot 8$ & 0.31 & $<0.00 \mathrm{I}$ \\
\hline II & 48 & 17 & 20.6 & $3 \cdot 4$ & 0.83 & NS - \\
\hline Other & 41 & 10 & $16 \cdot 6$ & $3 \cdot 1$ & 0.60 & $0.05 \subseteq$ \\
\hline \multirow{3}{*}{$\begin{array}{l}\text { Erythema nodosum } \\
\text { Present } \\
\text { Absent }\end{array}$} & & & & & & \\
\hline & 67 & 5 & 28.9 & $4 \cdot 0$ & 0.17 & $<0.00 \Omega$ \\
\hline & 116 & 35 & $49 \cdot 9$ & $5 \cdot 3$ & $0 \cdot 70$ & $0.00 \pi$ \\
\hline All patients & 183 & 40 & $78 \cdot 7$ & $6 \cdot 6$ & 0.51 & $<0.00 E$ \\
\hline
\end{tabular}


social groups.

Ninety four $(51.4 \%)$ patients had stage I sarcoidosis at presentation and 13 of these were smokers, compared with an expected number of 41.5 (table 2). Forty eight $(26.2 \%)$ patients had stage II and 34 $(18.6 \%)$ stage III sarcoidosis and in neither of these groups was there a significant difference between the number of smokers observed and that anticipated from the GHS figures, though when the stages were combined there were fewer smokers than expected $(p=0.03)$. Sixty seven $(36.6 \%)$ patients had been reported to have erythema nodosum around the time of presentation, 52 of these having stage I disease. Five of these patients with erythema nodosum were smokers, compared with an expected number of 28.9 .

Of the variables considered, only the stage of sarcoidosis and the presence or absence of erythema nodosum appeared to exert any influence on the magnitude of the association with non-smoking. Likelihood models were applied to test whether the apparent differences in the relative risks in the various subgroups were significant. Both stage (stage I $v$ other stages) and erythema nodosum (present or absent) considered in isolation were shown to have a significant effect on the magnitude of the relative risk from smoking (LR tests-stage: $\chi^{2}=8.9, \mathrm{p}=0.003$; erythema nodosum: $\chi^{2}=14.7, p<0.001$ ). When both variables were allowed simultaneously into a multiplicative model, the effect of erythema nodosum was still statistically significant at the $1 \%$ level but the statistical significance of stage was lost. The use of a more detailed model incorporating the four smoking categories gave a rather different picture. With a different relative risk of sarcoidosis allowed for within each smoking category, both stage (LR test: $\chi^{2}=16.9, \mathrm{p}<0.0001$ ) and erythema nodosum (LR test: $\chi^{2}=8 \cdot 3, p=0.004$ ) were again shown to modify these risks. This time, however, when the effect of both variables was considered simultaneously;

Table 2 Distribution of smoking habit in the different stages of sarcoidosis

\begin{tabular}{lclll}
\hline & Observed & Expected & $S E M$ & $O / E$ \\
\hline Non-smokers & 120 & 70.40 & 6.4 & 1.70 \\
Ex-smokers & 23 & 33.87 & 5.1 & 0.68 \\
Light smokers & 25 & 41.38 & 5.6 & 0.60 \\
Heavy smokers & 15 & 37.35 & 5.4 & 0.40 \\
Stage I $(n=94)$ & & & & \\
Non-smokers & 74 & 35.28 & 4.6 & 2.10 \\
Ex-smokers & 7 & 17.17 & 3.7 & 0.41 \\
Light smokers & 8 & 21.32 & 4.0 & 0.38 \\
Heavy smokers & 5 & 20.23 & 3.9 & 0.25 \\
Other stages $(n=89)$ & & & & \\
Non-smokers & 46 & 35.12 & 4.5 & 1.31 \\
Ex-smokers & 16 & 16.70 & 3.6 & 0.96 \\
Light smokers & 17 & 20.06 & 3.9 & 0.85 \\
Heavy smokers & 10 & 17.12 & 3.7 & 0.58 \\
\hline
\end{tabular}

Table 3 Estimated relative risks and $95 \%$ confidence intervals by stage and smoking categories

\begin{tabular}{lll}
\hline Smoking category & Stage I & Other stages \\
\hline Non-smokers & $1 \cdot 00$ & $1 \cdot 00$ \\
Ex-smokers & $0 \cdot 18(0 \cdot 10,0 \cdot 33)$ & $0.72(0 \cdot 43,1 \cdot 20)$ \\
Light smokers & $0 \cdot 17(0 \cdot 10,0 \cdot 30)$ & $0.68(0.40,1 \cdot 15)$ \\
Heavy smokers & $0 \cdot 11(0 \cdot 06,0 \cdot 21)$ & $0.44(0.24,0 \cdot 81)$ \\
\hline
\end{tabular}

erythema nodosum was found not to be of statistical significance while stage maintained significance at almost the $0 \cdot 1 \%$ level. Using likelihood models revealed no evidence of interaction between stage and erythema nodosum. The coefficients showing the relative risk in the ex-smokers, light smokers, and heavy smokers indicated the order that would have been expected from a "dose-response" relationship (table 3), though the difference between them was not significant (LR test: $\chi^{2}=2 \cdot 5, p=0.28$ ).

The paradox in the above analysis arises from the contribution of the ex-smokers and in particular the anomalous results in a small group of patients with erythema nodosum and more advanced stages of sarcoidosis. This group has five ex-smokers, compared with an expected number of $2 \cdot 1$, and their effect is critical. In the first analysis presented, where exsmokers and non-smokers were grouped together, the association between smoking and a lower incidence of sarcoidosis was stronger in patients with erythema nodosum, with differences between stage I and other stages possibly incidental $(p=0 \cdot 11)$. With exsmokers either considered separately, as in the second analysis, or grouped with smokers, the association appeared to be affected only by whether or not the patient had stage I disease. Comparison of the likelihood according to the alternative models showed a much better fit when ex-smokers were considered separately or included with smokers than when only current smoking was considered. The most simple model that describes the data adequately suggests that regular smoking at any time is associated with a lower incidence of sarcoidosis. Furthermore, this association is stronger in stage I disease, where the estimated relative risk compared with non-smokers is $0.15(95 \%$ confidence limits $0.09-0 \cdot 24)$ than in other stages, where the relative risk is $0.59(0.39-0.89)$.

\section{Discussion}

In this survey smoking was uncommon in patients with sarcoidosis. This was because a high proportion of patients had never smoked and also because there were fewer ex-smokers than expected. It could be argued that the recording of smoking habit in patients referred to hospital is different from a survey of the general population and that many of those patients 
recorded as non-smokers in this study were actually ex-smokers. There was, however, a difference in distribution of smoking habit between the different stages of sarcoidosis (table 2), implying that there was no consistent bias against the recording of exsmokers. Although retrospective analysis of clinical case records has many inherent shortcomings, our findings are consistent in different hospitals and throughout the period of study. In 19 patients there was no record of smoking habit, but even if all these patients had been smokers there would still have been significantly fewer patients smoking than would be expected. Sarcoidosis is frequently asymptomatic and intrathoracic disease is often discovered on a chest radiograph performed as a screening procedure. Nonsmoking habit, however, seems unlikely to have influenced referral for radiography in symptomless patients. This association between non-smoking and sarcoidosis persisted despite further analysis of sex and age distribution and socioeconomic grouping, factors known to influence smoking prevalence. We therefore believe that these results show that nonsmoking is more common in patients with sarcoidosis than in the general population.

The association between sarcoidosis and nonsmoking was greatest in patients with stage I and much less for those with the other stages of the disease. A further study would be required to see whether smoking alters the progression of sarcoidosis from stage I to more extensive pulmonary disease. Erythema nodosum also initially appeared to exert an influence on the magnitude of the association but this did not persist after further statistical analysis. A study of patients with erythema nodosum with or without sarcoidosis would be necessary to discover whether non-smoking is also associated with this condition.

The results of our survey contrast with those reported from Canada by Warren, ${ }^{1}$ who found that the smoking habits of 75 patients with sarcoidosis were similar to those expected from the general population figures for the Prairie Region for 1973, 31 $(41 \%)$ being current smokers. The mean age of these Canadian patients was, however, higher (42 years) and all 75 had been diagnosed as having sarcoidosis with compatible histological appearances in lymph node or liver biopsy specimens. Many patients with stage I disease may therefore have been excluded, which could have increased the percentage of smokers in this group of patients. A case controlled study of 141 patients with sarcoidosis in the State of Georgia in the United States, which included those found to have hilar adenopathy on routine chest radiography, showed a highly significant negative association among white patients between sarcoidosis and cigarette smoking, but this was not found in the black population. ${ }^{4}$ Similarly, a study from New York City reported that, of 240 patients with sarcoidosis confirmed by either Kveim test or other typical histo- $\frac{\bar{\sigma}}{\bar{c}}$ logical appearances, $113(47 \%)$ had never smoked $\vec{\sigma}$ cigarettes, compared with only $62(26 \%)$ of 240 con-으 trol patients with tuberculosis matched for age, sex, race, and area of residence. ${ }^{5}$

The reasons why non-smoking is associated with sarcoidosis are unknown. Smoking has been shown tow hasten the clearance of inhaled particles from the lung $^{6}$ and, in vitro, alveolar macrophages from smok- $x$ ers are more active than those from non-smokers. ${ }^{7 \pm}$ Hence the lungs of smokers may be able to remove $\vec{\circ}$ the antigen or other stimulus that might otherwise go on to produce the histological changes of sarcoidosis. Alternatively, cigarette smoke may suppress $\mathrm{T}$ lym-음 phocyte function ${ }^{8}$ and therefore prevent $T$ cell activa- tion, which is thought to precede the formation of aO granuloma. These hypotheses, however, do not: explain why those who have stopped smoking for at least six months have a reduced risk of developing $\overrightarrow{\vec{\theta}}$ sarcoidosis. Whatever the mechanism, our survey suggests that non-smoking is commoner in patients. with sarcoidosis than in the general population and that further studies are needed to see whether smoking habit has any influence on the progression oro severity of this disorder.

We wish to thank Miss A Hunter for typing the $\overrightarrow{\vec{B}}$ manuscript.

\section{Appendix: Model for estimation of relative risk}

Let the probability that a non-smoker has sarcoidosis be denoted by $\lambda_{0}$ (which will remain unknown), and let the relative risk in ex-smokers, light smokers and $\tilde{x}$ heavy smokers be denoted $r_{E}, r_{L}$ and $r_{H}$ respectively. Then the corresponding probabilities of having sarcoidosis in these three categories will be $\lambda_{0} r_{E}, \lambda_{0} r_{L}, \frac{\delta}{2}$ and $\lambda_{0} r_{\mathrm{H}}$.

We will let $P_{N}, P_{E}, P_{L}$, and $P_{H}$ denote the proba- 9 bilities that an individual is a non-smoker, ex-smoker, $\rightarrow$ light smoker, and heavy smoker. These terms will in fact depend on the age and sex of the subject and the N year to which we are referring and will be obtained from the General Household Survey.

Prob (non-smoker and diseased) $=P_{N} \lambda_{0}$

Prob (ex-smoker and diseased) $=P_{E} \lambda_{0} r_{E}$

Prob (light smoker and diseased) $=P_{L} \lambda_{0} r_{L}$

Prob (heavy smoker and diseased) $=\mathbf{P}_{\mathbf{H}} \lambda_{0} \mathbf{r}_{\mathbf{H}}$

Hence, given that a patient has sarcoidosis, we can 0 write down the conditional probability for each of the

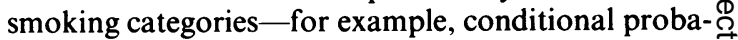
bility of being an ex-smoker $=P_{E} r_{E} / d$, where $d=P_{N} \stackrel{\mathbb{Q}}{\Omega}$ $+P_{E} r_{E}+P_{L} r_{L}+P_{H} r_{H}$.

Consequently, by using the usual maximum like- 
lihood approach we can obtain estimates and standard errors for the relative risk terms in the above expression. Thus we choose estimates for $r_{E}, r_{L}$, and $\mathrm{r}_{\mathrm{H}}$ to maximise

$$
\begin{aligned}
& \mathrm{L}=\Sigma \log \left(\mathrm{p}_{\mathrm{N}} / \mathrm{d}\right)+\Sigma \log \left(\mathrm{p}_{\mathrm{E}} \mathrm{r}_{\mathrm{E}} / \mathrm{d}\right)+ \\
& \text { non- ex- } \\
& \text { smokers smokers } \\
& \begin{array}{cl}
+\underset{\operatorname{light}}{\sum \log \left(\mathrm{p}_{\mathrm{L}} \mathrm{r}_{\mathrm{L}} / \mathrm{d}\right)}+\underset{\text { heavy }}{\sum \log \left(\mathrm{p}_{\mathrm{H}} \mathrm{r}_{\mathrm{H}} / \mathrm{d}\right)} \\
\text { smokers } & \text { smokers }
\end{array}
\end{aligned}
$$

and obtain standard errors for these estimates from the matrix of second partial derivatives of $\mathrm{L}$ with respect to $r_{E}, r_{L}$, and $r_{H}$.

This model can readily be extended to test whether these relative risks differ significantly for various subgroups (for example, men versus women), and whether they differ according to the stage of disease.

To illustrate the approach let us consider whether the relative risks are the same in stage I disease as in other stages. If we define a dummy variable $s$ to take the value 1 when a patient has stage I disease, and to take the value 0 otherwise, then we could simply follow the approach described above but replace $r_{E}$ throughout by $r_{E} m_{E}^{s}, r_{L}$ by $r_{L} m_{L}^{s}$, and $r_{H}$ by $r_{H} m_{H}{ }^{s}$. In this model $r_{E}, r_{L}$, and $r_{H}$ denote the relative risks in stages II onwards, while $\mathrm{m}$ indicates the extent to which these relative risks are multiplied in stage I disease. A particularly interesting model in practice has been this model with the restriction

$$
\mathrm{m}_{\mathrm{E}}=\mathrm{m}_{\mathrm{L}}=\mathrm{m}_{\mathrm{H}} \text {. }
$$

The statistical significance of terms in the model is obtained by using likelihood ratio tests. Confidence limits for the relative risks are obtained via a logarithmic transformation: that is, the logarithms of the relative risks and the associated standard errors are estimated and used to obtain confidence limits in the logarithmic scale. These are then transformed back to confidence limits for relative risk.

\section{References}

1 Warren CPW. Extrinsic allergic alveolitis: a disease commoner in non-smokers. Thorax 1977;32:567-9.

2 Harries AD, Baird A, Rhodes J. Non-smoking: a feature of ulcerative colitis. Br Med J 1982;284:706.

3 Office of Population Census and Surveys. General Household Survey 1982. Cigarette Smoking 1972-82. (OPCS Monitor No 3.) London: HMSO, 1983.

4 Comstock GW, Keltz H, Spencer DJ. Clay eating and sarcoidosis. A controlled study in the State of Georgia. Am Rev Respir Dis 1961;84(suppl):130-4.

5 Terris M, Chaves AD. An epidemiological study of sarcoidosis. Am Rev Respir Dis 1966;94:50-5.

6 Albert RE, Peterson HT Jr, Bohning DE, Lippman M. Short-term effects of cigarette smoking on bronchial clearance in humans. Arch Environ Health 1975;30:361-7.

7 Ando M, Sugimoto M, Nishi R, et al. Surface morphology and function of human pulmonary macrophages from smokers and non-smokers. Thorax 1984;39:850-6.

8 Razman TL, Elliot LH, Rogers AS. Suppression of lymphocyte function by products derived from cigarette smoke. Am Rev Respir Dis 1975;111:453-7. 